\title{
La basilique civile de Nysa du Méandre
}

\section{Serdar Hakan Öztaner}

\section{OpenEdition}

\section{Journals}

Édition électronique

URL : http://journals.openedition.org/anatoliaantiqua/389

DOI : 10.4000/anatoliaantiqua.389

Éditeur

IFEA

Édition imprimée

Date de publication : 1 mai 2016

Pagination : 311-320

ISBN : 9782362450648

ISSN : 1018-1946

\section{Référence électronique}

Serdar Hakan Öztaner, "La basilique civile de Nysa du Méandre », Anatolia Antiqua [En ligne], XXIV | 2016, mis en ligne le 11 décembre 2018, consulté le 16 février 2021. URL : http://

journals.openedition.org/anatoliaantiqua/389; DOI : https://doi.org/10.4000/anatoliaantiqua.389 


\section{ANATOLIA ANTIQUA ESKI ANADOLU}

\section{XXIV}

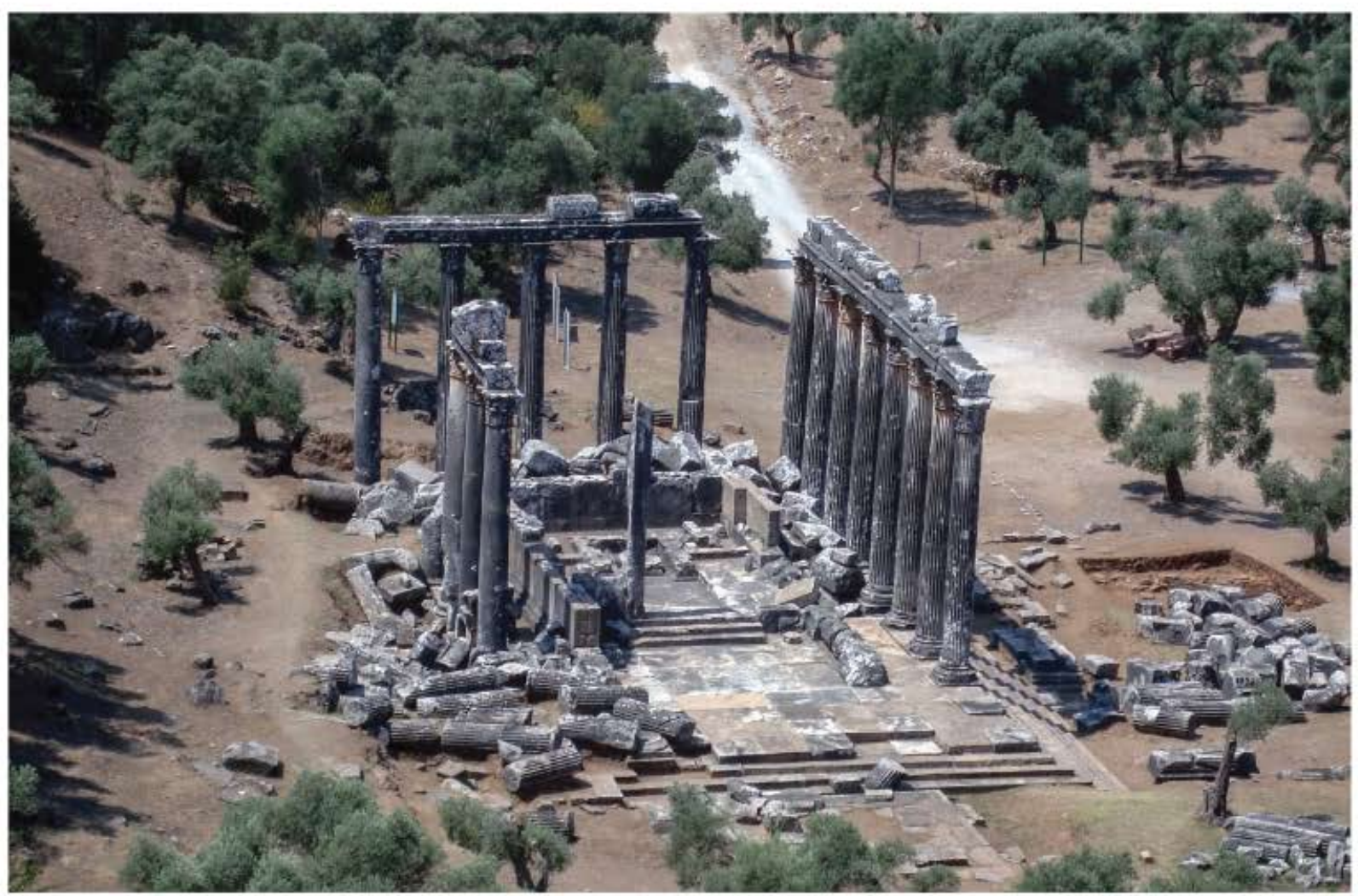

INSTITUT FRANÇAIS D'ETUDES ANATOLIENNES GEORGES-DUMEZIL

CNRS USR 3131

DE BOCCARD 


\section{TABLE DES MATIERES}

Ergul KODAS,

Le surmodelage du crâne au Néolithique au Proche-Orient : techniques de surmodelage et

expérimentations

Isabella CANEVA et Eric JEAN,

Mersin-Yumuktepe : une mise au point sur les derniers travaux

Turan EFE et Bérengère PERELLO,

Second Millenium site distribution and pottery of Inland Northwestern Anatolia

Antoine PEREZ,

Amida 6 : Antiochos IV, le "Hanigalbat" et la Sophène

Ergün LAFLI et Hadrien BRU,

Inscriptions et monuments funéraires gréco-romains d'Anatolie occidentale

Oğuz TEKİN et Aliye EROL-ÖZDİZBAY,

Coins from Allianoi excavations: Campaign of 2001

Nuran ŞAHIN,

Etude iconographique des monnaies autonomes frappées par Colophon-sur-Mer :

nouveaux acquis

Vera SAUER,

Konventionelle Individualität. Zur Münzprägung nordanatolischer Städte in der römischen

Kaiserzeit

Oğuz TEKIN,

Balance weights in the collection of the Anatolian Civilizations Museum in Ankara

\section{CHRONIQUES DES TRAVAUX ARCHEOLOGIQUES EN TURQUIE 2015}

Çiğdem MANER,

Preliminary report on the third season of the Konya-Ereğli Survey (KEYAR) 2015

Dominique BEYER, Isabelle CHALIER et Françoise KIRNER,

Rapport préliminaire sur les travaux de la mission archéologique de Zeyve Höyük-Porsuk 2015 ...

Sami PATACI et Ergün LAFLI,

Field surveys in Ardahan in 2015

Jean-Charles MORETTI avec la collaboration de Nicolas BRESCH, Isabel BONORA,

Jean-Jacques MALMARY et Olivier RISS,

Claros, le temple d'Apollon : travaux réalisés en 2015

Serdar Hakan ÖZTANER,

La basilique civile de Nysa du Méandre

Abuzer KIZIL, Koray KONUK, Patrice BRUN, Laurent CAPDETREY, Raymond DESCAT,

Pierre FROHLICH, Didier LAROCHE, Enora LE QUERE, Francis PROST, Baptiste VERGNAUD,

Eurômos : rapport préliminaire sur les travaux réalisés en 2015

O. HENRY et E. ANDERSON, Chr. BOST, Ö. ÇAKMAKLI, F. CEDERLING, A. COMMITO,

M. CORMIER-HUGUET, A. COUTELAS, A. DOLEA, D. ERGENÇ, A. FRECCERO,

A. FREJMAN, P. LEBOUTEILLER, F. LESGUER, D. LÖWENBORG, V. LUNGU,

Fr. MARCHAND-BEAULIEU, A. SITZ, P. DE STAEBLER, B. VERGNAUD, 


\section{Serdar Hakan ÖZTANER*}

\section{LA BASILIQUE CIVILE DE NYSA DU MEANDRE}

Le bâtiment sujet de cet article se trouve à Nysa du Méandre, actuellement aux frontières de Sultanhisar/Aydın, et se situe au Sud-Est du théâtre vers le bouleuterion (Gerontikon) et l'agora (Fig. 1). C'est en 1994, lors de l'élargissement de la route moderne conduisant au village de Kavaklı, que le bâtiment a été découvert. Ont été alors mis au jour un chapiteau figuré ainsi que des éléments architecturaux supposés appartenir à une basilique civile ${ }^{1}$.

Les caractéristiques de l'édifice et ses fonctions architecturales ont, par la suite, été confirmées par les investigations scientifiques conduites sur le bâtiment lui-même et sur ses abords entre 2012 et $2015^{2}$. L'ouverture d'une route sur une largeur de $4,85 \mathrm{~m}$, en traversant la partie est de l'édifice, a détruit, après observation, l'angle nord-est du mur externe du bâtiment (Fig. 2), tandis que les fouilles ont permis de constater que le réservoir des sanitaires modernes, situé au Nord et construit à l'intérieur de l'édifice sur des éléments architecturaux, a détruit une partie du mur nord externe de celui-ci (Fig. 3).

\section{DESCRIPTION}

Les murs extérieurs ont une épaisseur d'environ $1,2 \mathrm{~m}$ et sont construits de mortier de chaux et de moellons selon la technique de l'opus incertum. La longueur du mur ouest est mesurée à $14,85 \mathrm{~m}$ à l'extérieur et à 13,6 m à l'intérieur. L'extrémité sud du mur ouest a été construite avec de grands blocs de conglomérat, et sa hauteur, constituée de trois rangées in situ, est de 2,2 m (Fig. 4). Dans le même temps et toujours dans le même axe, le mur est de l'édifice, qui se trouve au bord de la route moderne, est également, à une de ses extrémités constitué de blocs de conglomérat. La longueur du mur nord est de $23 \mathrm{~m}$ à l'extérieur et de 20,4 m à l'intérieur. Ce- pendant, la route moderne passant sur l'édifice n'a permis d'observer sa longueur que sur 15,5 m. Dans la partie est du mur nord, à $12 \mathrm{~m}$ de l'angle interne nord-ouest, a été mise au jour une ouverture correspondant à une porte de $1,50 \mathrm{~m}$ de large. Cette ouverture a été ultérieurement condamnée par un mur de moellons composé de matériaux de réemploi comprenant notamment le pied d'un kline en forme de tête de panthère et une base en marbre.

Les murs précédemment mentionnés forment un espace rectangulaire de 20,4 x 13,6 m. Le stylobate est conservé dans la partie sud. Sur ce stylobate, mis au jour sur 15,56 m de long, la présence d'éléments architecturaux (17 blocs en marbre) appartenant à des piliers suggère que l'édifice s'ouvrait vers le Sud. A l'Ouest du stylobate, a été découvert, in situ, une base de pilier latéral $(1,09 \times 0,60 \mathrm{~m}$ de largeur et $0,33 \mathrm{~m}$ de hauteur) adossée à l'extrémité du mur ouest (Fig. 4-5). Ce pilier latéral situant 3,94 m à l'Est compte deux bases de piliers in situ $(1,12 \mathrm{x}$ 1,99 $\mathrm{m}$ de largeur, 0,39 $\mathrm{m}$ de hauteur) espacées de 7,04 m (Fig. 5). Nos recherches ont permis de constater que ces deux bases supportaient deux piliers composés chacun de quatre blocs de marbre (soit huit blocs au total) et mesuraient avec leur bases 5,13 m. (Fig. 11). Nous avons déduit de l'emplacement des blocs des piliers que ces derniers étaient tombés vers le Nord à la suite d'un tremblement de terre, à l'exception du bloc in situ de la première rangée de piliers à l'Est (Fig. 6). Sur la base du pilier latéral ouest se trouve toujours un bloc de marbre in situ dont manque la partie supérieure $(0,92 \times 0,48 \mathrm{~m}$ de largeur, $1,26 \mathrm{~m}$ de hauteur conservée). A l'Est, l'autre pilier latéral comprenait un bloc de marbre qui mesure $0,92 \times 0,48 \mathrm{~m}$ de largeur et $2,44 \mathrm{~m}$ de hauteur intacte et qui a été retrouvé à $25 \mathrm{~m}$ au Nord-Est de l'édifice. Les traces laissées sique.

*) Université d'Ankara, Faculté des Lettres et d'Histoire-Géographie, Département d'archéologie, Section d'archéologie clas-

1) Idil $1999: 51$.

2) Les fouilles de Nysa en 2012-2015 ont été dirigées par S.H. Öztaner, Doç. Dr., sous la direction du Musée d’Aydın. 


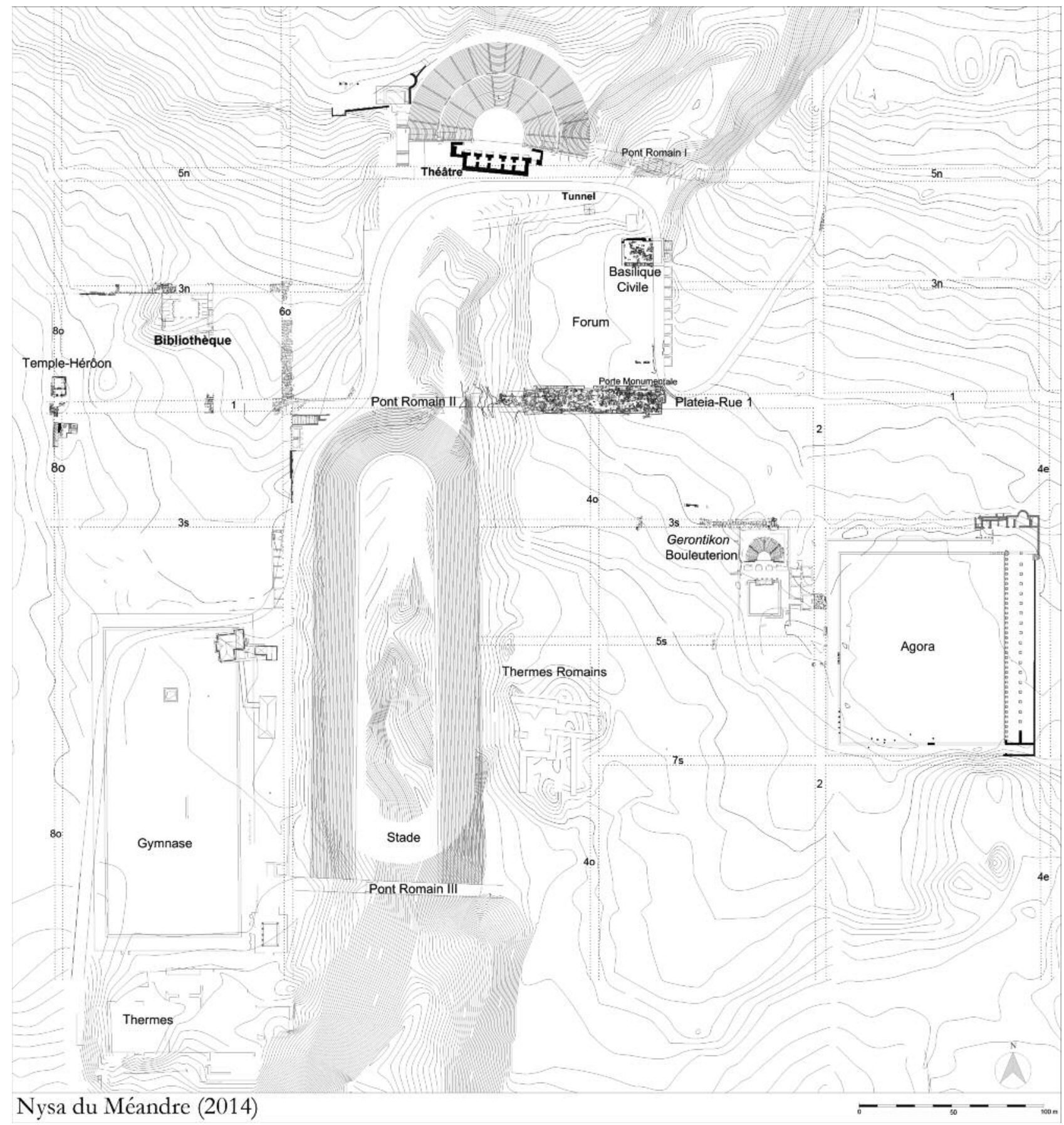

Fig. 1 : Plan de Nysa du Méandre (2014). 


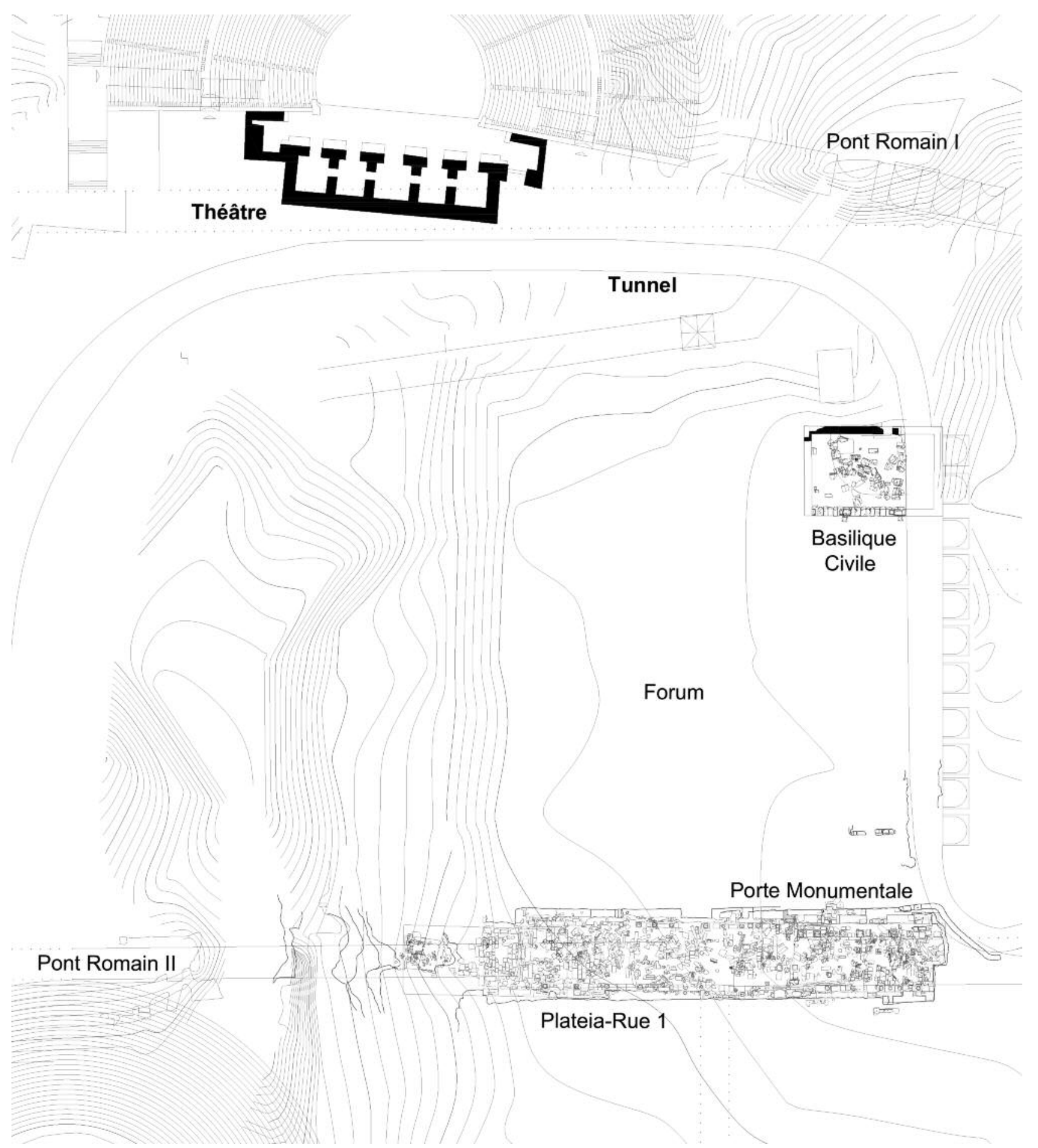

Fig. 2 : Plan de la place du forum de Nysa. 


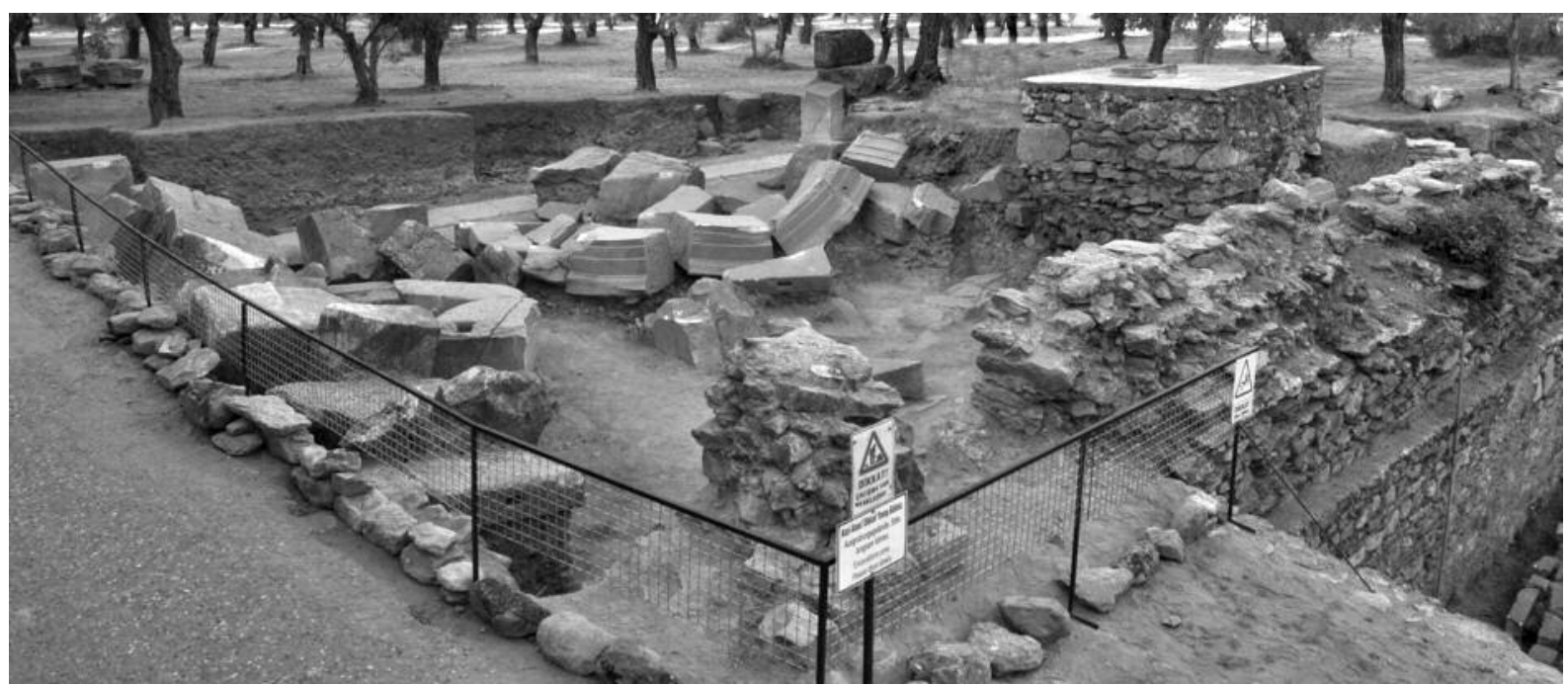

Fig. 3 : Vue de la basilique civile vers le Sud-Ouest (2012).

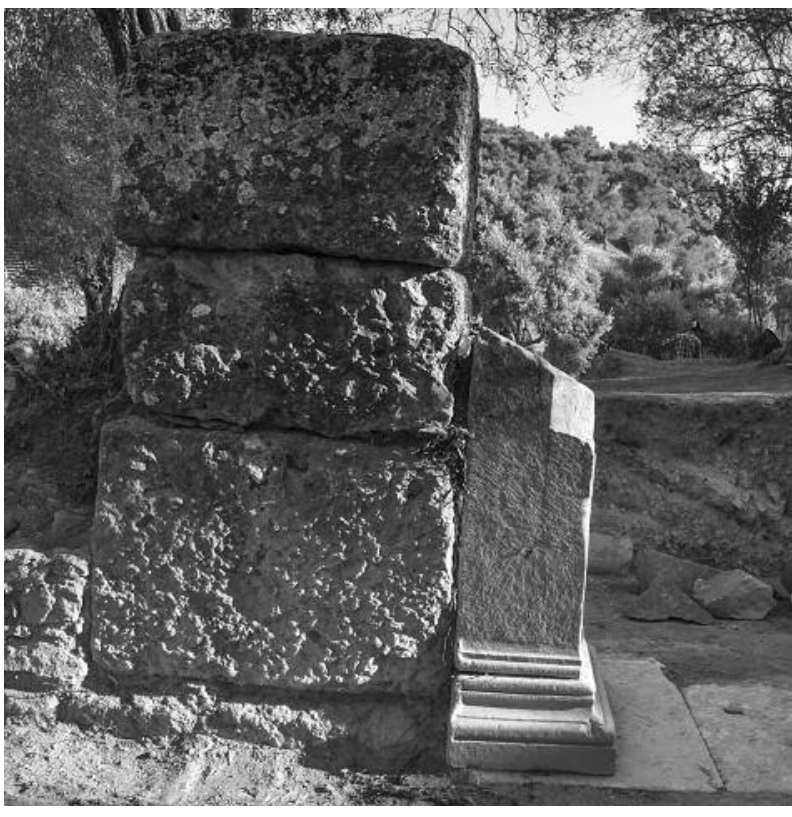

Fig. 4 : L'extremité du mur ouest et le pilier latéral ouest.

sur le bloc par l'utilisation d'engin de construction montrent que ce dernier a été arraché et traîné au moment de la construction de la route moderne.

Le chapiteau pilastre $(0,80 \times 0,49 \mathrm{~m}$ de largeur à la base, $0,46 \mathrm{~m}$ de hauteur intacte), que nous avons trouvé près du pilier est, peut appartenir au pilier latéral est. Le long des bases des trois faces de ce chapiteau on retrouve un ornement de kymation ionique. Au-dessus de cette rangée d'ornements on observe, au centre et sur les côtés, des motifs de feuille d'acanthe. Entre les feuilles d'acanthe, les kaulis, les hélix ainsi que des rinceaux de feuilles sont représentés symétriquement. Les volutes sont cassées (Fig. 7). Le chapiteau pilastre de l'autre pilier latéral ouest n'existe plus.

On a constaté que les piliers centraux est et ouest étaient surmontés de chapiteaux figurés (Fig. 8-11). D'après nos recherches le chapiteau figuré $(1,82 \mathrm{~m} \times 1,45 \times 0,70 \mathrm{~m}$ de hauteur) mis au jour près du pilier aurait été découvert en 1994 (Fig. 9), ce dernier devait surmontait le pilier est. Quant aux autres fragments des chapiteaux figurés, ils appartiennent au pilier ouest. La composition de ces deux chapiteaux figurés peut être comprise, malgré le coin endommagé du chapiteau du pilier est, et ce à partir de l'observation du chapiteau ouest. (Fig. 10).

Ainsi, au-dessus d'un rang de feuilles d'acanthe, on observe sur les faces avant des petits côtés des chapiteaux, le monstre marin Scylla avec ses pattes de chien au centre, et sur les extrémités un triton jeune, et un plus âgé, avec leur buste humain et leur queue de poisson. Les espaces vides sont occupés par des motifs figurant des poissons. Sur les longs côtés, des Néréides évoluent sur des queues de triton et, à l'arrière-plan, des Eros chevauchent des dauphins.

Ces chapiteaux figurés ressortissent au même type iconographique que l'on observe sur ceux de la basilique civile de Magnésie du Méandre ${ }^{3}$ et que l'on désigne sous le nom de "chapiteau Scylla"4. Nous pouvons suggérer que ces deux chapiteaux sont l'œuvre du même atelier de sculpture.

3) Öztaner $2012: 167-187$.

4) Bingöl $1992: 418-423$. 


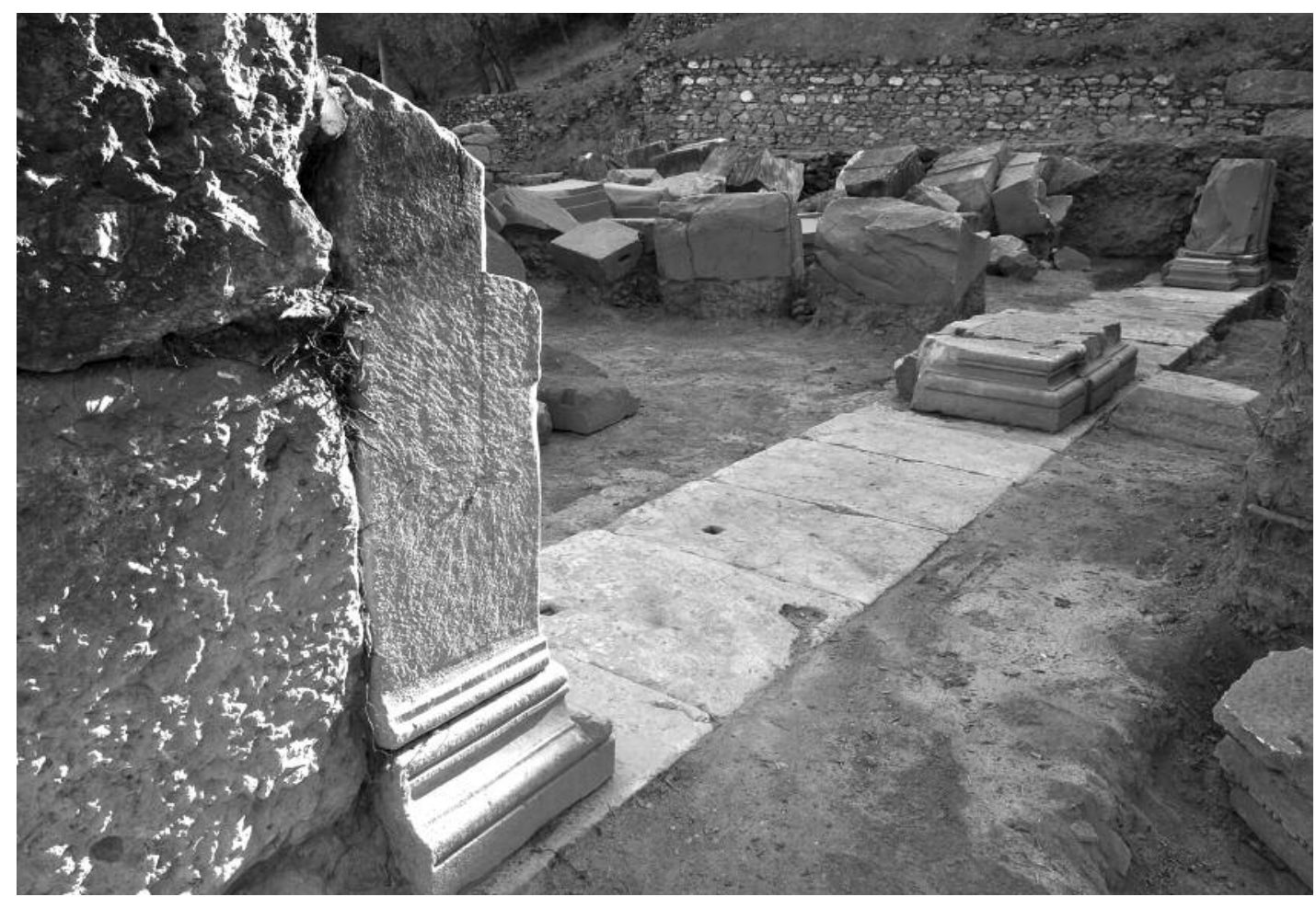

Fig. 5 : Les piliers (vue vers l'Est).

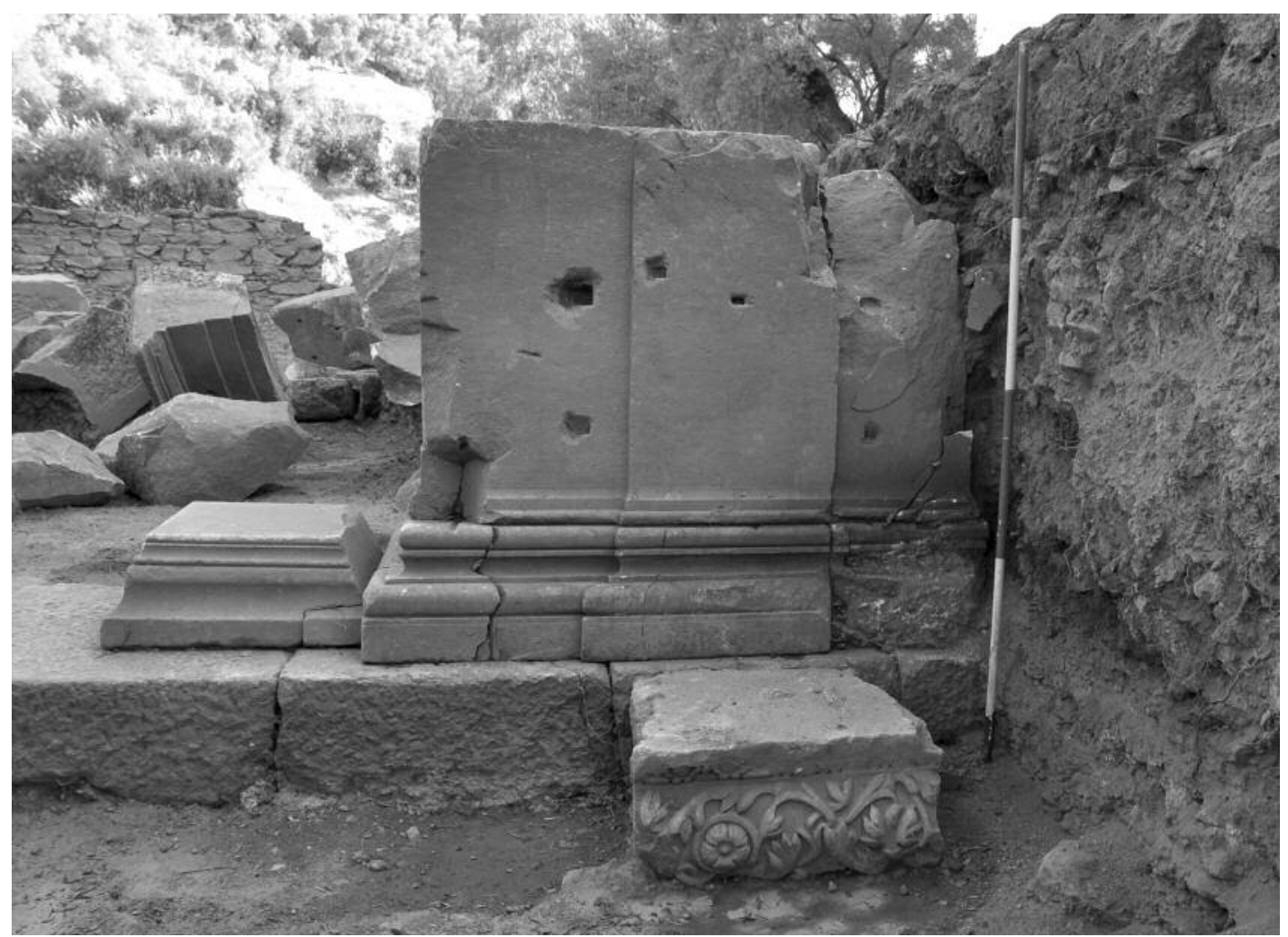

Fig. 6 : Le pilier situé à l'Est. 


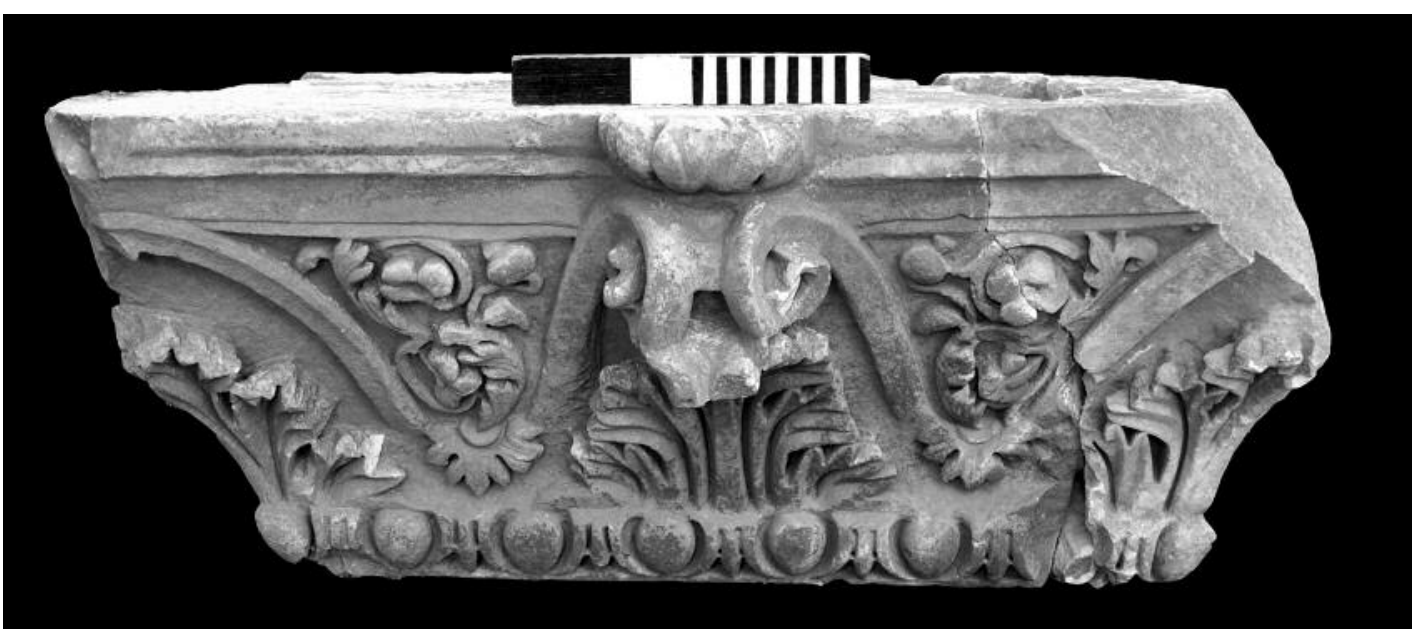

Fig. 7 : Le chapiteau pilastre.

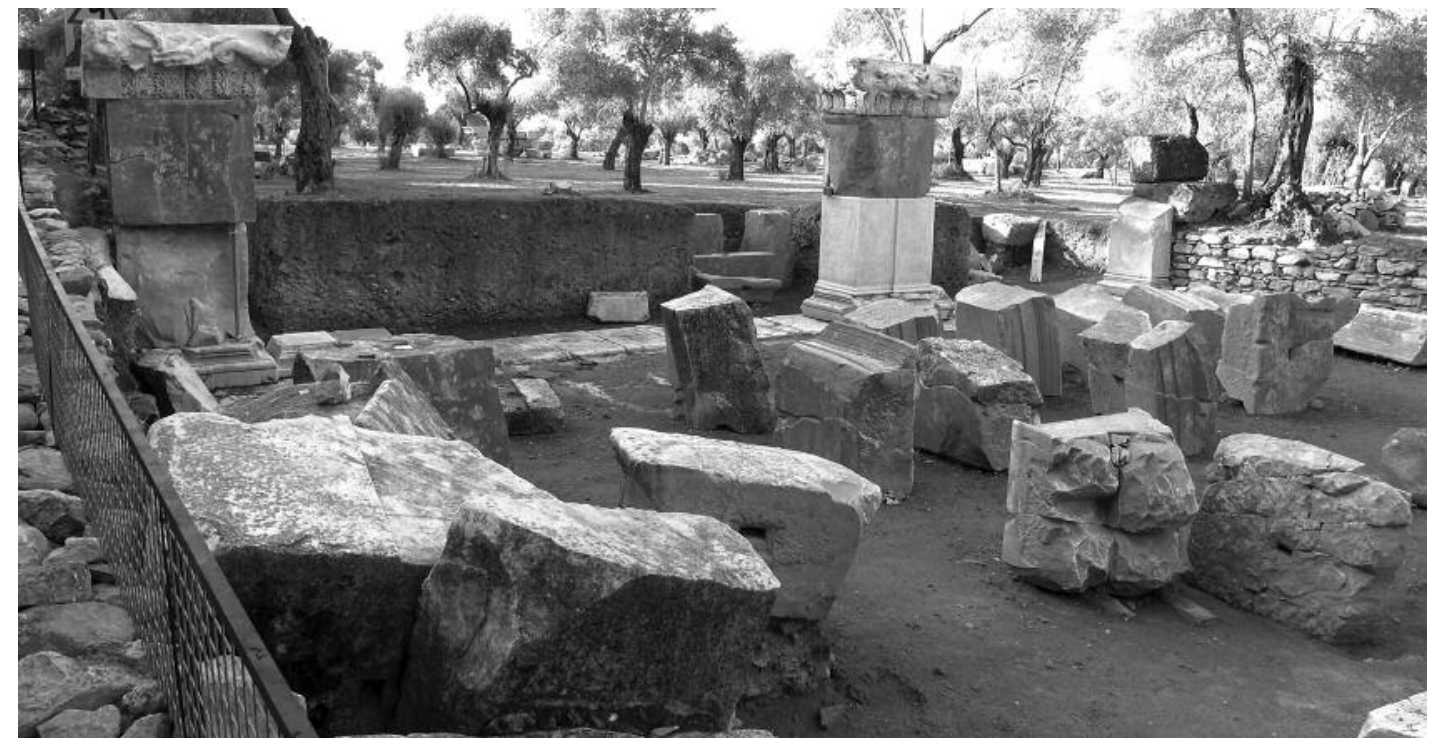

Fig. 8 : Vue de la basilique en 2014 après anastylose.

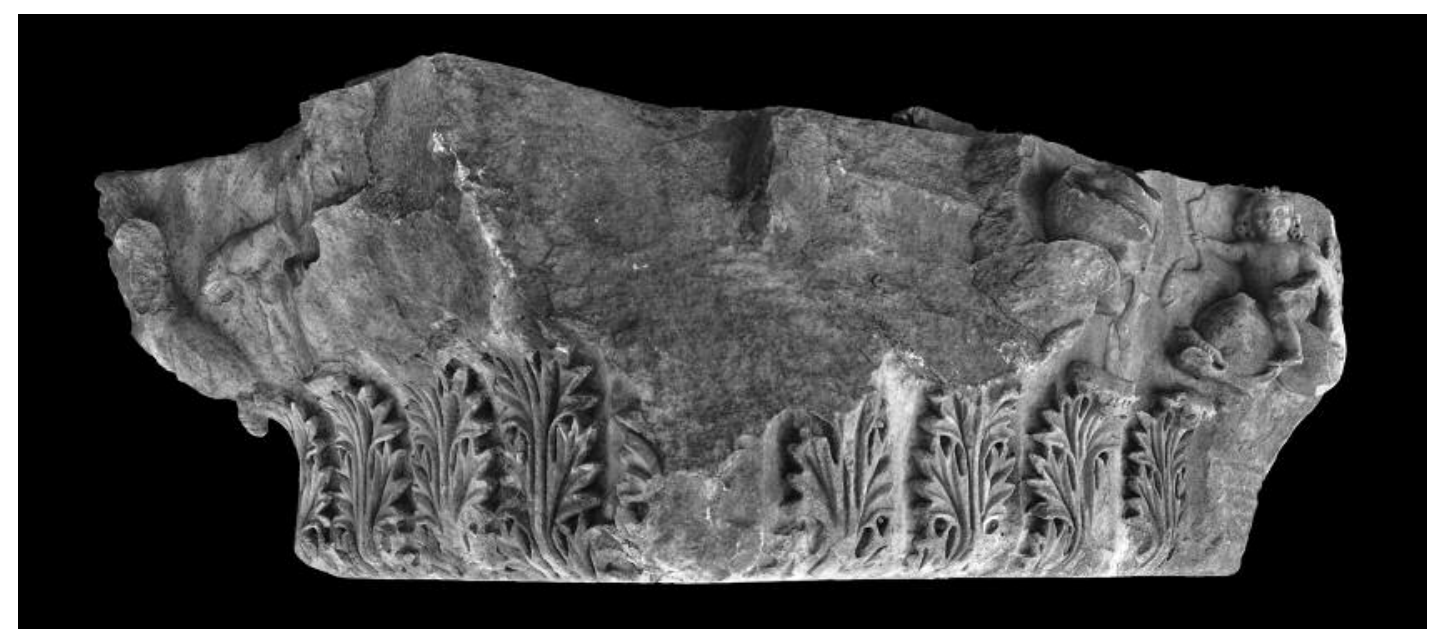

Fig. 9 : Le chapiteau figuré du pilier est. 


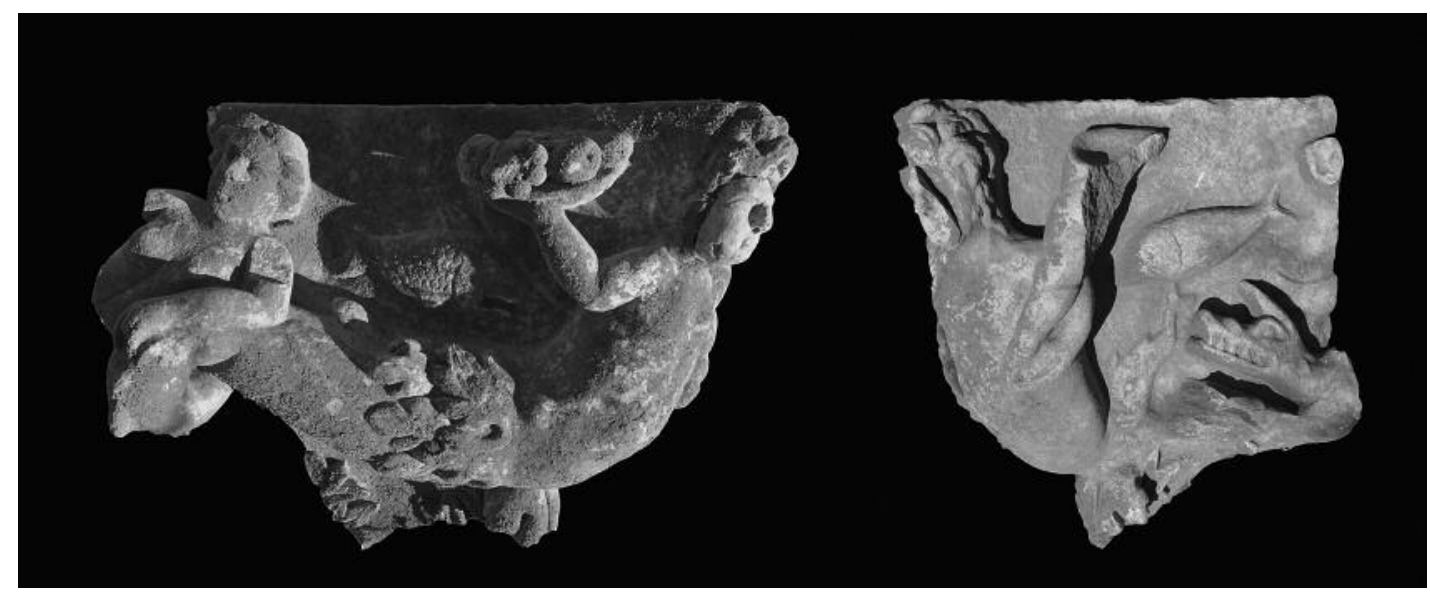

Fig. 10 : Le chapiteau figuré du pilier ouest.

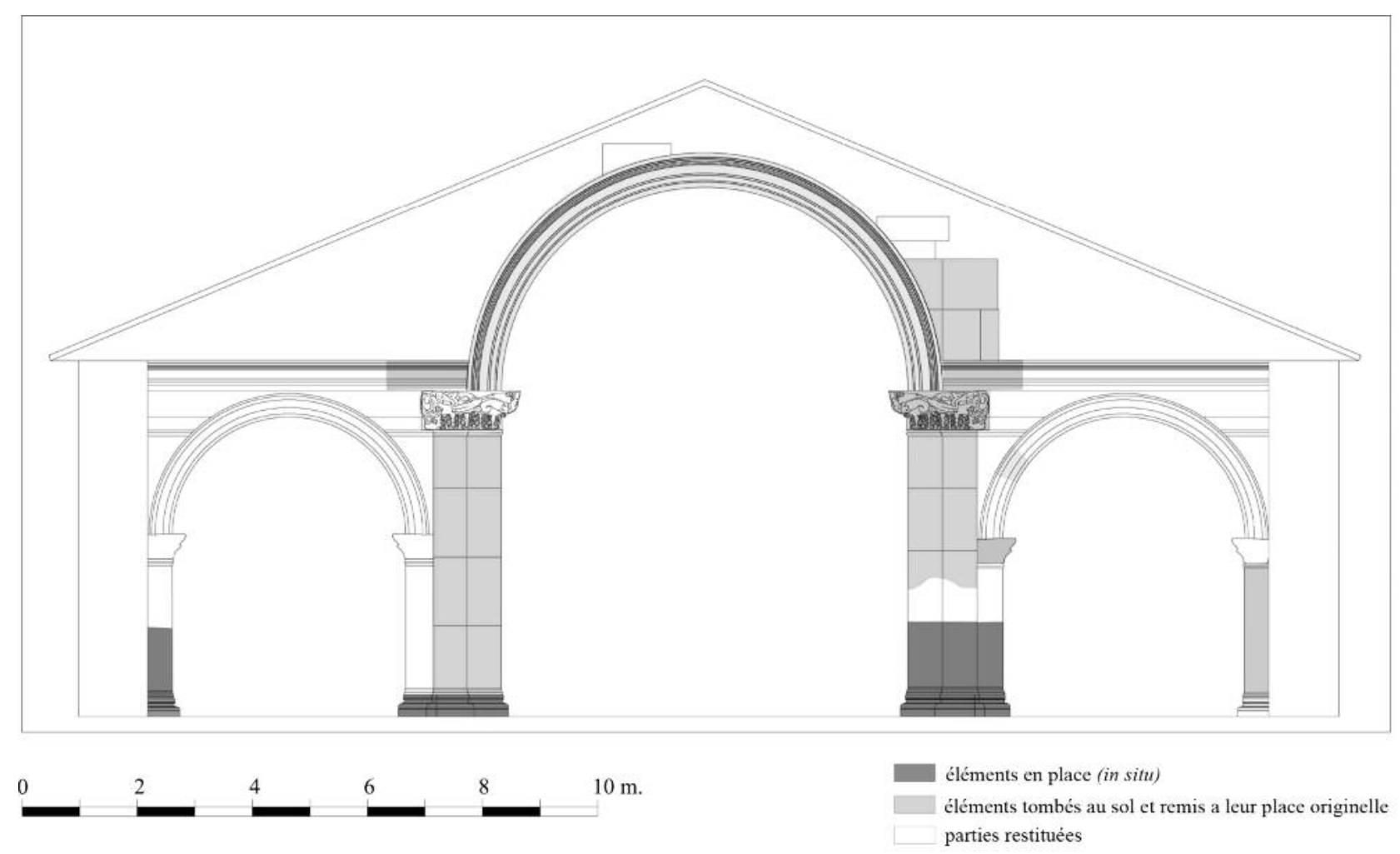

Fig. 11 : Restitution de la façade de la basilique civile.

Lors des fouilles, parmi les éléments architecturaux de l'édifice, et qui en représentent un groupe important, 15 claveaux appartenant à trois arcs surmontés par quatre piliers ont été retrouvés. Ces piliers se composaient de quatre rangées de blocs que coiffaient les chapiteaux figurés. Ils supportaient l'arc central dont nous mesurons le diamètre à 7,3 m, et se situaient à environ $5,8 \mathrm{~m}$ de hauteur. D'après la restitution proposée, la clé de l'arc central se serait élevée à une hauteur de 9,4 m. Les chapiteaux de pilastre, sur les faces arrières des piliers centraux est et ouest et les piliers latéraux, portaient les arcs latéraux de 4,16 m de diamètre. Toujours d'après la restitution, les arcs latéraux se tenaient au niveau de $3,23 \mathrm{~m}$ et s'élevaient jusqu'à 5,3 m (Fig. 11).

\section{DATATION}

Les éléments et les ornements architecturaux (comme les feuilles d'acanthe) de l'édifice, ainsi que leurs caractéristiques, montrent qu'ils appartiennent à la seconde moitié du deuxième siècle de 
notre ère. A été mis au jour le pavement en marbre de l'édifice qui est partiellement conservé. A $0,30 \mathrm{~m}$ au-dessus de ce pavement, une couche brûlée a été observée : elle contient des blocs de marbre transformé en calcaire et un nombre important de clous en bronze. Cette couche correspond à l'effondrement du toit de l'édifice consécutif à un grand incendie (Fig. 6). Cependant, une couche d'environ 0,50 à $0,60 \mathrm{~m}$ de débris de terre retrouvée immédiatement au-dessus de la couche brûlée, nous laisse supposer que l'écroulement des blocs de l'édifice est intervenu longtemps après l'incendie.

La position des éléments architecturaux de l'édifice indique un important séisme avec une direction Nord-Sud ayant entraîné l'écroulement des blocs vers le Nord. Pour le moment il n'a pas été possible de déterminer l'année du séisme car les céramiques retrouvées dans les couches archéologiques n'ont pas subi de datation stratigraphique.

\section{FONCTION DE L'EDIFICE}

Nous n'avons rencontré aucune trace appartenant à des éléments architecturaux lors des sondages effectués, sur $4 \mathrm{~m}$, le long des axes des piliers. Cependant, en 2013, les recherches géophysiques réalisées sur le périmètre de la terrasse où l'édifice prend place, dans sa zone nord-est, ont permis d'obtenir des résultats importants qui laissent supposer que l'édifice s'allongeait vers le Sud avec une rangée de colonnade. De plus, le long des côtés sud et ouest de cette terrasse, les résultats géophysiques laissent supposer que des murs entouraient celle-ci. En conséquence c'est un forum, d'environ 70 x $80 \mathrm{~m}$ de dimensions, que nous avons proposé de restituer et dans lequel l'édifice occupait le côté est (Fig. 12).

En 2013 d'autres résultats archéologiques o nt confirmé la présence de ce forum. Une structure d'environ $66 \mathrm{~m}$ au Sud des piliers a été mise au jour, ainsi qu'une porte monumentale de $23 \mathrm{~m}$ de long qui permettait d'accéder à la zone du forum supposé Cette porte monumentale est placée sur la voie principale de Nysa, voie que nous avons appelée "Plateia-Rue 1"6 (Fig. 12-13). Celle-ci, ainsi mise au jour du côté est de la ville, avait été supposée être, lors des fouilles précédentes, à l'Ouest de la cité. A Nysa les voies mises au jour sont en général pavées de pierres de conglomérat, ce qui n'est pas le cas de la partie est où se situe la porte monumentale composée de dalles en calcaire. La partie de la voie côté est, est composée de colonnades de 9,5 m de largeur, elle passe par le centre de la cité, part du pont romain central et continue vers l'Est en délimitant le côté sud de la zone du forum.

D'après les données obtenues en fouilles, les recherches géophysiques et les caractéristiques architecturales de l'édifice, nous pouvons dire que nous sommes en présence d'une basilique civile qui se situe sur le long côté du forum. Son architecture et les chapiteaux figurés qui l'ornaient sont semblables à ceux de la basilique civile de Magnésie du Méandre, ce qui va bien dans le sens de l'identification d'une basilique civile pour le bâtiment de Nysa.

Les basiliques civiles ${ }^{7}$ de l'époque romaine se situent généralement au centre des villes, liées directement au forum. Ce sont des bâtiments juridiques ${ }^{8}$ et commerciaux conçus pour abriter des conditions météorologiques. Elles sont allongées et présentent habituellement un plan à trois nefs séparées par deux rangées de colonnes.

Nous supposons être dans ce cas de figure avec l'édifice de Nysa, c'est-à-dire en présence d'une basilique civile avec un tribunal au Nord $(20 \times 15$ m). Ajoutons que de la même façon, en Anatolie occidentale, à Ephèse ${ }^{9}$, à Smyrne ${ }^{10}$, à Hiérapolis ${ }^{11}$, à Xanthos $^{12}$, à Cremna ${ }^{13}$, à Aspendos ${ }^{14}$ les basiliques civiles se situent, comme la basilique de Nysa, inscrite dans ce même modèle appelé "stoa basilique", le long côté des fora.

S.H.Ö.

5) Öztaner $2014: 225-245$.

6) Kadıŏlu $2011: 108$; 2014. Öztaner : $2014: 225-245$.

7) Pour les basiliques civiles Boëthius et Ward-Perkins 1970 : 127-131 ; Crema 1959 : 167-171, 370-375, $515-521$; Gros 1994 : 612-616 ; Gros 1996 : 235-261 ; Ginouvès $1994: 207-216$; Müller $1937: 250-261$.

8) David $1983: 219-241$.

9) Alzinger 1974 ; Fossel-Peschl 1982.

10) Naumann et Kantar $1950: 69-114$.

11) Andria et Rossignani $2012: 127-152$.

12) Cavalier $2012: 189-199$.

13) Mitchell $1989: 229-245$.

14) Balty $1991: 411-412$; Lanckoronski 1890 : 96-98. 


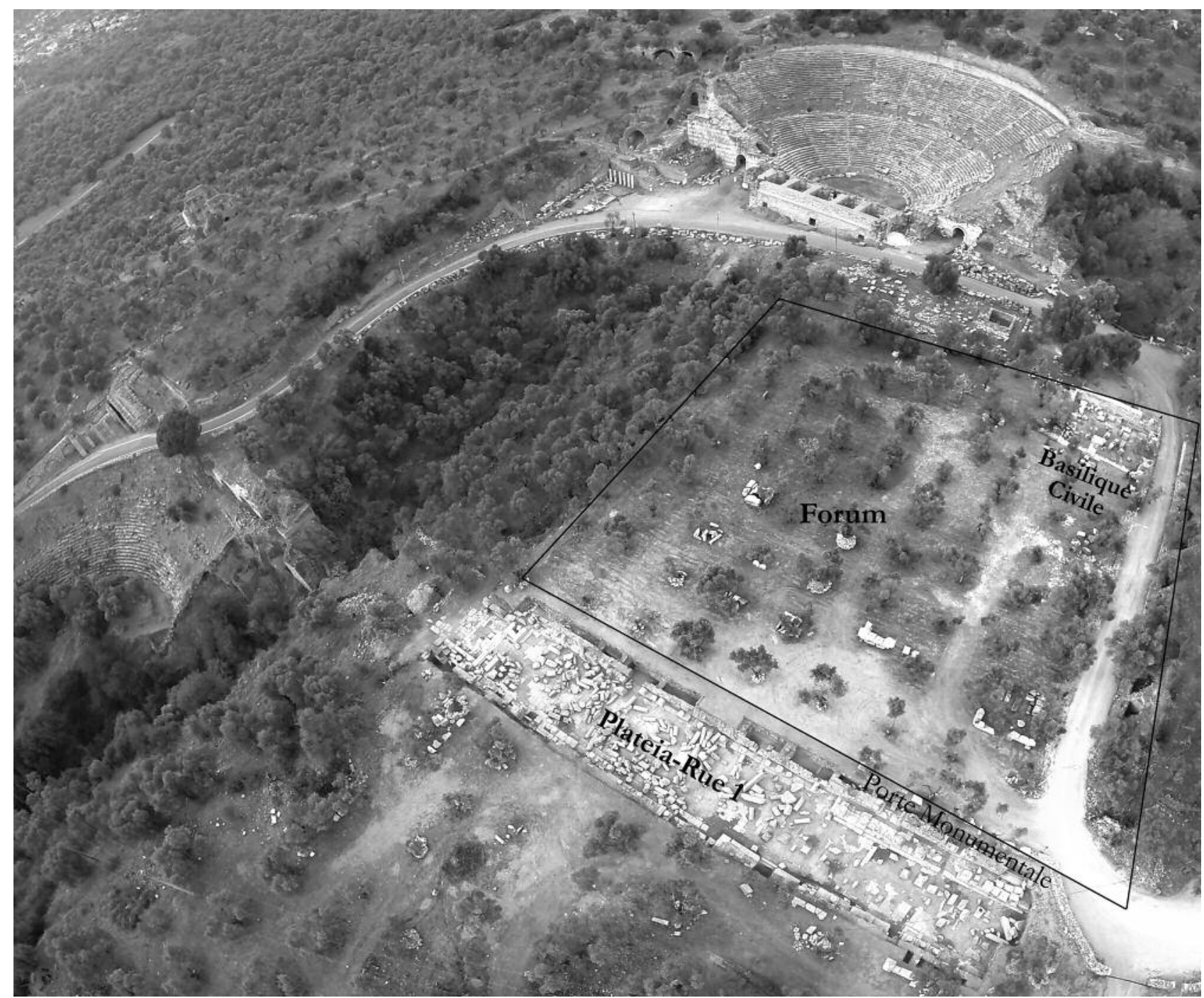

Fig. 12 : Vue aerienne de la place du forum.

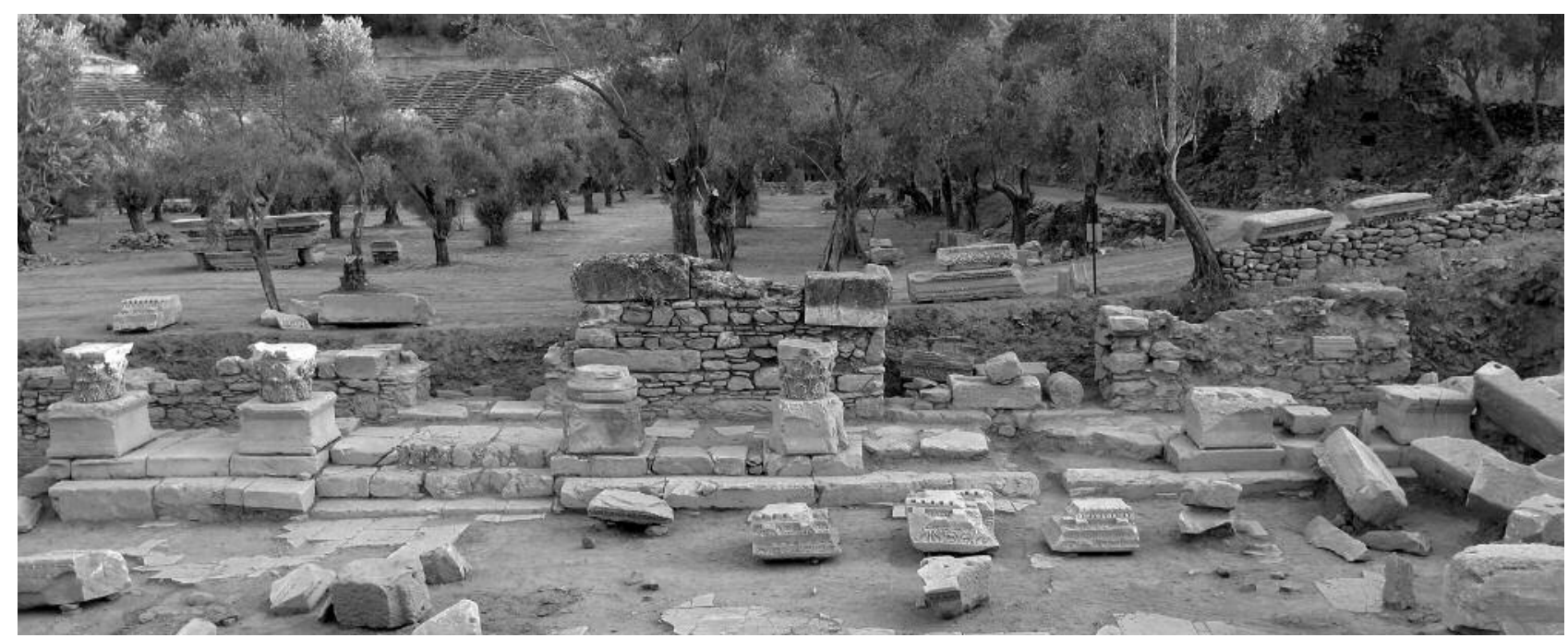

Fig. 13 : Porte monumentale du forum. 


\section{BIBLIOGRAPHIE}

Alzinger, W., 1974 : Augusteische Architektur in Ephesos, Wien.

Andria, F. et Rossignani M.P., 2012 : "La stoabasilique de Hiérapolis de Phrygie. Architecture et contexte urbain", in Cavalier, L., Descat, R. et des Courtils J. (éds.), Basiliques et Agoras de Grèce et d'Asie Mineure, Ausonius Editions, M 27 : 127-152.

Balty, J.-Ch., 1991 : Curia Ordinis, Recherches d'architecture et d'urbanisme antiques sur les curies provinciales du monde romain, Bruxelles : 411-412.

Bingöl, O., 1992 : "Das Skyllakapitell von Magnesia am Mäander”, in Fronig, H., Hölscher, T. et Mielsch Kotinos, H. (éds.), Festschrift für Erika Simon, Philipp von Zabern : 418-423.

Boëthius, A. et Ward-Perkins, J.B., 1970 : Etruscan and Roman Architecture, London.

Crema, L., 1959 : “Architettura Romana”, Enciclopedia Classica, XII,3,1.

Cavalier, L., 2012 : "La basilique civile de Xanthos", in Cavalier, L., Descat, R. et des Courtils J. (éds.), Basiliques et Agoras de Grèce et d'Asie Mineure, Ausonius Editions, M 27 : 189-199.

David, J.-M., 1983 : "Le tribunal dans la basilique. Evolution fonctionnelle et symbolique de la république à l'empire", Architecture et société, de l'archaïsme grec à la fin de la république romaine, Actes du colloque international organisé par le Centre national de la Recherche scientifique et l'Ecole française de Rome, Rome, 2-4 décembre 1980, Rome : 219-241.

Fletcher, B., 1956 : A History of Architecture on the Comparative Method, $16 \mathrm{t}^{\mathrm{h}}$ ed., London.

Fossel-Peschl, E.A, 1982 : Die Basilica am Staatmark in Ephesos, Graz.
Ginouvès, R., 1994 : “Aux origines de la Basilique”, Tranquillitas, Mélanges en l'honneur de Tran tam Tinh, Québec.

Gros, P., 1994 : "Basilica” EAA : 612-616.

- 1996 : L'Architecture Romaine 1. Les Monuments publics, Paris.

İdil, V., 1999 : Nysa ve Akharaka, İstanbul, Yaşar Eğitim ve Kültür Vakfi.

Kadığlu, M., 2011 : "Vorbericht Über Die Arbeiten im Gerontikon von Nysa am Mäander (2006-2009)", JDAI 126 : 107-154.

- 2014 : Das Gerontikon von Nysa am Mäander. Forschungen in Nysa am Mäander Band 3, Mainz, Zabern.

Lanckoronski, K., 1890 : Städte Pamphyliens und Pisidiens I, Wien : 96-98.

Mitchell, S., 1989 : "The Hadrianic Forum and Basilica at Cremna", in Başgelen, N. et Lugal, M. (éds.), Festchrift für Jale Inan, İstanbul : 229-245.

Müller, V., 1937, "The Roman Basilica", AJA 41 : 250-261.

Naumann, R. et Kantar, S., 1950 : "Die Agora von Smyrna, Kleinasien und Byzanz", IstForsch 17 : 69-114.

Öztaner, S.H., 2012 : "La Basilique Civile de Magnésie du Méandre", in Cavalier, L., Descat, R. et des Courtils J. (éds.), Basiliques et Agoras de Grèce et d'Asie Mineure, Ausonius Editions, M 27 : 167-187.

Öztaner, S.H., Akdoğu, A.E.N. et Gökalp, Ö.N., 2014 : "Nysa Ad Maeandrum'daki Yeni Bulgular Üzerine Bir Değerlendirme", in Aşkit, Ç., Kalaycıŏulları, S., Kayapınar, R., Üstünel Keyinci, C. et Öztürk, R. (éds.), In Memoriam Filiz Öktem, A.Ü. DTCF, Ankara : 225245. 


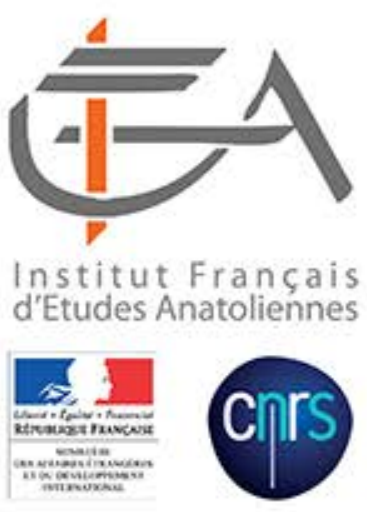

Eurômos : le temple de Zeus vu de l'Est (cliché Sönmez Alemdar).

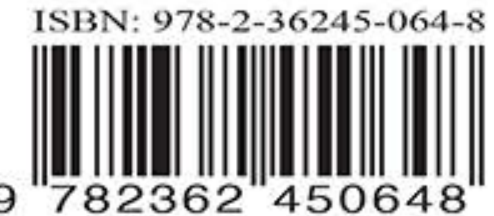

